\title{
Polaritonic Feshbach resonance
}

\author{
N. Takemura ${ }^{1 \star}$, S. Trebaol' ${ }^{1}$ M. Wouters' ${ }^{2}$, M. T. Portella-Oberli' and B. Deveaud ${ }^{1}$
}

\begin{abstract}
A Feshbach resonance occurs when the energy of two interacting free particles comes into resonance with a molecular bound state. When approaching this resonance, marked changes in the interaction strength between the particles can arise. Feshbach resonances provide a powerful tool for controlling the interactions in ultracold atomic gases, which can be switched from repulsive to attractive ${ }^{1-4}$, and have allowed a range of many-body quantum physics effects to be explored ${ }^{5,6}$. Here we demonstrate a Feshbach resonance based on the polariton spinor interactions in a semiconductor microcavity. By tuning the energy of two polaritons with anti-parallel spins across the biexciton bound state energy, we show an enhancement of attractive interactions and a prompt change to repulsive interactions. A mean-field two-channel model quantitatively reproduces the experimental results. This observation paves the way for a new tool for tuning polariton interactions and to move forward into quantum correlated polariton physics.
\end{abstract}

A semiconductor microcavity is a unique system where exciton-polaritons emerge from the strong coupling between an exciton and a photon. The demonstration of Bose-Einstein condensation of exciton-polaritons in a semiconductor microcavity $^{7}$ has attracted much attention and opened a wide field of research on polariton quantum fluids, such as superfluidity ${ }^{8}$, quantum vortices $^{9}$ and Bogoliubov dispersion ${ }^{10-12}$. Many more examples could be proposed to highlight the fact that polaritons provide a concrete realization of a bosonic interacting many-body quantum system, complementing the work performed on ultracold atom systems.

Furthermore, polaritons exhibit a polarization degree of freedom, with a one-to-one connection to two counter circular polarizations for their photonic part. The different excitonic content of both polarization states results in asymmetric spinor interactions. Such spinor interactions offer a wide range of effects and a very rich physics to explore in semiconductor microcavities ${ }^{13-18}$.

In this work, we demonstrate a Feshbach resonance in a polariton semiconductor microcavity. Feshbach biexcitonic resonant scattering is investigated through spectrally resolved circularly polarized pump-probe spectroscopy on a III-V based microcavity (Methods). To bring the energy of a two-lower polariton state into resonance with the biexciton state we change the cavity exciton detuning (Fig. 1a,c). We evidence the resonant polariton scattering by probing the anti-parallel spin polariton interactions when scanning the two-polariton energy across the bound biexciton state. We clearly show the enhancement of polariton interactions and the change of their character from attractive to repulsive. Moreover, we observe a decrease of the polariton resonance amplitude when the lower polariton energy is in the vicinity of the biexciton energy. The results are modelled by numerical simulations based on a meanfield two-channel model that includes coupling between polaritons and biexcitons as a key ingredient. It is worth mentioning that several works ${ }^{19-22}$ have already reported observations of coupling between polaritons and biexcitons without reaching the regime of the Feshbach resonance reported here.

The expected signatures of the Feshbach resonance phenomenon are twofold. First, a strong variation of the strength and sign of the scattering amplitude; second, a reduction of the free particle density through the coupling with the molecular bound state. Both are sensitive to the energy difference between the free particles and the molecular states. These two energy states refer, in our system, to the state of two anti-parallel spin lower polaritons and the biexciton state, respectively.

We investigate both experimental signatures of the Feshbach resonance. This coherent effect requires working in the coherent regime, therefore we set a zero delay between pump and probe (results from experiments with different pump-probe delays are presented in Supplementary Methods). For a given pump power, we measure through the transmitted probe beam the energy shift and the amplitude variation of the lower polariton resonance induced by the presence of the polariton population generated by the pump (Fig. 1b,d,e). From negative to positive cavity detuning, the energy of the two lower polaritons (2LP, one from the $\sigma_{+}$polariton cloud created by the pump, and one $\sigma_{-}$polariton coming from the probe) ranges from below to above the molecular biexciton state energy, passing through the resonance (Fig. 1c). In Fig. 2a, we plot the lower polariton energy shift versus exciton-cavity detuning for a polariton density of $5.1 \times 10^{10}$ polaritons $\mathrm{cm}^{-2}$ generated by the pump. This result clearly shows a dispersive shape, characteristic of the resonant scattering. Indeed, in dilute atomic gas systems the Feshbach resonances are evidenced by a dispersive shape of the scattering length at resonance ${ }^{3}$. In these atomic systems, the dispersive shape diverges in the case of magnetic Feshbach resonances with very long molecular lifetime ${ }^{1,3}$. Here, however, a smooth dispersive shape shows up, similar to the case of optical Feshbach resonances with finite molecular lifetime $e^{3,4}$. For polaritons, the finite lifetime of the molecular biexciton state prevents the dispersive shape form diverging at the resonance. Note that the sign and amplitude of the energy shift are related to the character and strength of the polariton interaction respectively. Our result directly shows that, at resonance, the energy shift switches from redshift to blueshift, demonstrating the drastic modification of the interaction character from attractive to repulsive. This measured shape provides clear evidence for a Feshbach resonance.

In Fig. 2b, we plot the variation of the polariton resonance amplitude as a function of the cavity detuning. This result shows the resonant conversion of two polaritons with anti-parallel spins into a biexciton. The simultaneous observations of the change of the polariton interaction character from attractive to repulsive together with the enhanced polariton loss through the coupling with the molecular biexciton state gives direct evidence for the polaritonic Feshbach resonant scattering. The resonance effect shown in Fig. 2a,b is located at an exciton-cavity detuning energy

${ }^{1}$ Laboratory of Quantum Optoelectronics, EPFL, CH-1015 Lausanne, Switzerland, ${ }^{2}$ TQC, University of Antwerp, Universiteislpein 1, 2610 Antwerpen, Belgium. *e-mail: naotomo.takemura@epfl.ch 
a

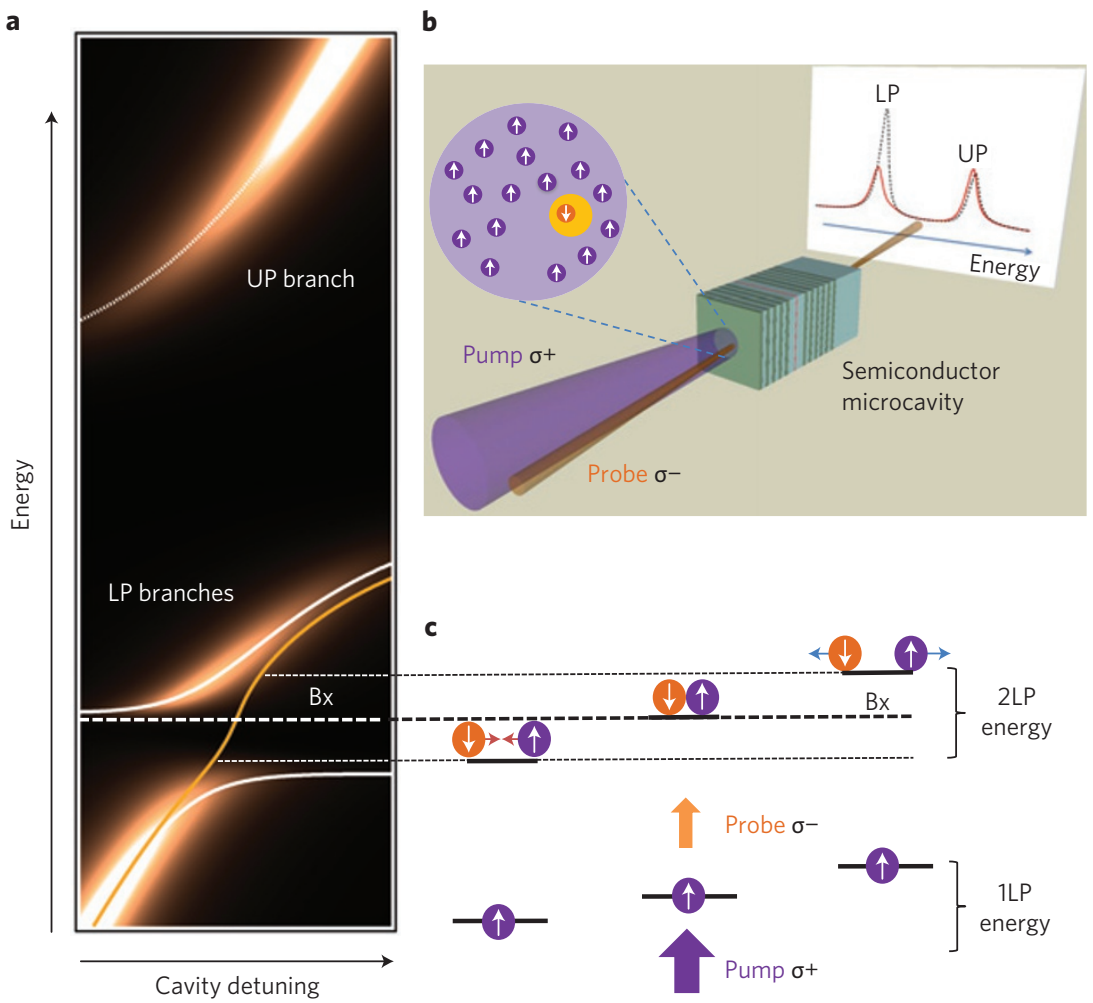

d
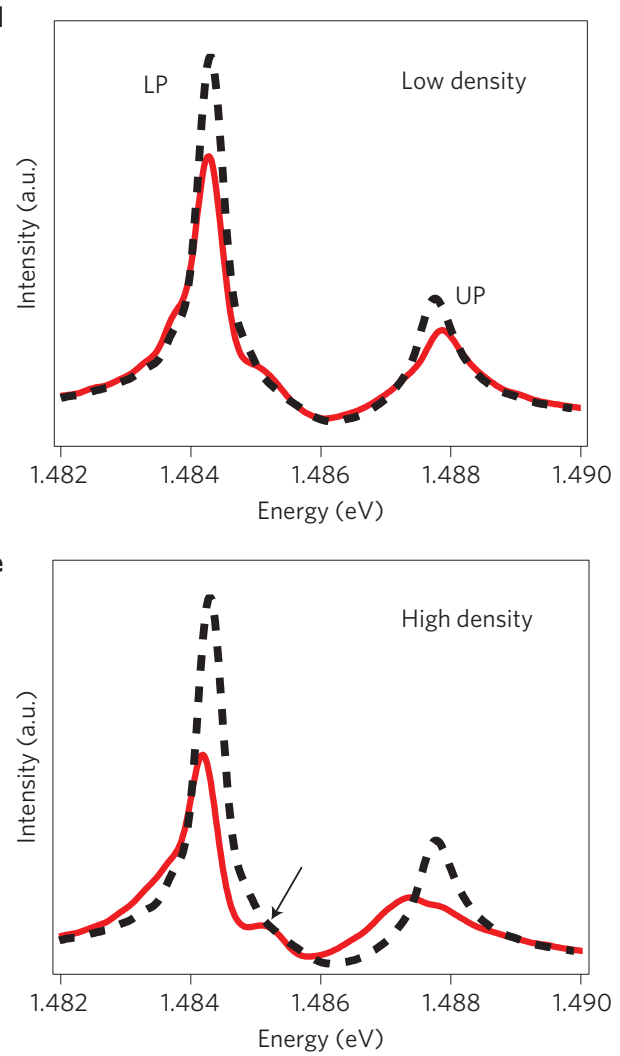

Figure 1 | Experimental scheme used for the polaritonic Feshbach resonance. a, The spin-down polariton energy varies as a function of the cavity detuning in the presence of a spin-up polariton population. In the perturbative regime, the lower polariton resonance shows a dispersive shape (orange line) around the crossing point with the biexciton energy state (bold dashed line). At higher polariton spin-up densities, the lower polariton branch splits into two at the biexciton crossing energy (solid white lines). This effect results from the strong coupling with the biexciton (Bx) resonance. UP and LP branches stand for the upper and lower polariton resonances respectively. $\mathbf{b}$, A pump beam creates a gas of spin-up polaritons (violet) and the probe introduces a few spin-down polaritons (orange), which interact with the gas. Inset $\mathbf{b}$ shows the transmission spectrum of the probe $\sigma-$ without (black line) and with (red line) the presence of a spin-up polariton gas. c, Two-polariton energy with respect to the biexciton state determines the interaction configuration: attractive (red arrows) or repulsive (blue arrows). The resulting polariton energy is depicted in Fig. 1a. d,e, Pump-probe spectra for polariton densities of $4.5 \times 10^{10} \mathrm{~cm}^{-2}(\mathbf{d})$ and $1.5 \times 10^{11} \mathrm{~cm}^{-2}(\mathbf{e})$, Cavity detuning is $-1.1 \mathrm{meV}$. In e, the black arrow indicates the appearance of a new resonance resulting from the strong coupling between the LP branch and the biexciton.

of $0.5 \mathrm{meV}$, which corresponds to the expected energy range where the two-lower polariton and the biexciton energies overlap (the green region in Fig. 2). This resonance is located $1.5 \mathrm{meV}$ below the exciton energy, in agreement with measured biexciton spectral properties of the same sample previously reported in ref. 20.

To highlight the physics behind our observations, we have performed numerical simulations based on a two-channel model, which includes coupling between polariton and biexciton $\left(g_{\mathrm{BX}}\right)$ in addition to the normal mode coupling between exciton and photon $\left(\Omega_{X}\right)$. For our system, it is given by the following Hamiltonian ${ }^{23,24}$ :

$$
\begin{aligned}
H= & \Omega_{X}\left(a_{c \sigma} \psi_{X \sigma}^{+}+a_{c \sigma}^{+} \psi_{X \sigma}\right)+\frac{U_{\mathrm{bg}}}{2}\left(\psi_{X \sigma}^{+} \psi_{X-\sigma}^{+} \psi_{X-\sigma} \psi_{X \sigma}\right) \\
& +g_{\mathrm{BX}}\left(\psi_{\mathrm{BX}}^{+} \psi_{X \sigma} \psi_{X-\sigma}+\psi_{\mathrm{BX}} \psi_{X \sigma}^{+} \psi_{X-\sigma}^{+}\right)
\end{aligned}
$$

Here, $a_{c \sigma}$ and $\psi_{X \sigma}\left(a_{c \sigma}^{+}\right.$and $\left.\psi_{X \sigma}^{+}\right)$are photon and exciton annihilation (creation) operators. The polarization state of the photon and the exciton are defined by the spin variable $(\sigma,-\sigma)$ as $(\uparrow, \downarrow)$. The coefficient $g_{\mathrm{BX}}$ represents the scattering coupling between two antiparallel spin polaritons and one biexciton. The underlying physical picture is the coupling of two polaritons to the biexciton bound state through the Coulomb interaction of their excitonic content. A background interaction between anti-parallel spin polaritons is represented by $U_{\mathrm{bg}}$. This interaction is found to be attractive (Supplementary Methods). After a mean-field approximation, an effective quadratic Hamiltonian $H_{\text {eff }}$ for the spin-down probe polariton and the biexciton amplitude is represented by the following $3 \times 3$ matrix:

$$
H_{\mathrm{eff}}=\left[\psi_{X \downarrow} a_{c \downarrow} \psi_{B}\right]^{+}\left[\begin{array}{ccc}
\varepsilon_{X}+U_{\mathrm{bg}} n_{X \uparrow}-i \frac{\gamma_{X}}{2} & \Omega_{X} & g_{\mathrm{BX}} \sqrt{n_{X \uparrow}} \\
\Omega_{X} & \varepsilon_{c}-i \frac{\gamma_{c}}{2} & 0 \\
g_{\mathrm{BX}} \sqrt{n_{X \uparrow}} & 0 & \varepsilon_{B}-i \frac{\gamma_{B}}{2}
\end{array}\right]\left[\begin{array}{c}
\psi_{X \downarrow} \\
a_{c \downarrow} \\
\psi_{B}
\end{array}\right]
$$

where the density $n_{\mathrm{X} \uparrow}$ corresponds to the excitonic content of the spin-up polariton density $n_{\mathrm{P}}$ generated by the pump. $\varepsilon_{X}, \varepsilon_{c}, \gamma_{B}, \gamma_{c}$ and $\gamma_{X}$ refer to the exciton energy, cavity photon energy, biexciton linewidth, photon linewidth and exciton homogeneous linewidth, respectively. Note that the polaritonic Feshbach resonance is determined by the polariton-biexciton scattering term $g_{\mathrm{BX}}$ that describes microscopically how the excitonic content of the two anti-parallel spin polaritons interact resonantly with the biexciton state. By analogy to the Feshbach resonance in atomic system, this term corresponds to the coupling between the open and closed channels ${ }^{3}$. A discussion about the direct coupling between the cavity mode and the biexciton state is presented in the Supplementary Methods. Numerical results give the $\sigma$-probe spectra, from which we can extract the energy position and the amplitude of polariton resonances for $n_{\mathrm{p}}=0$ and $n_{\mathrm{p}} \neq 0$, and thus their energy shift and amplitude variation. 


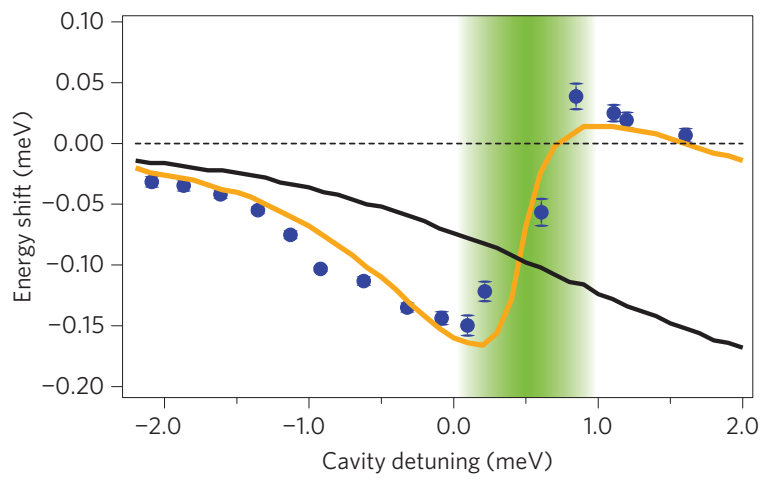

b
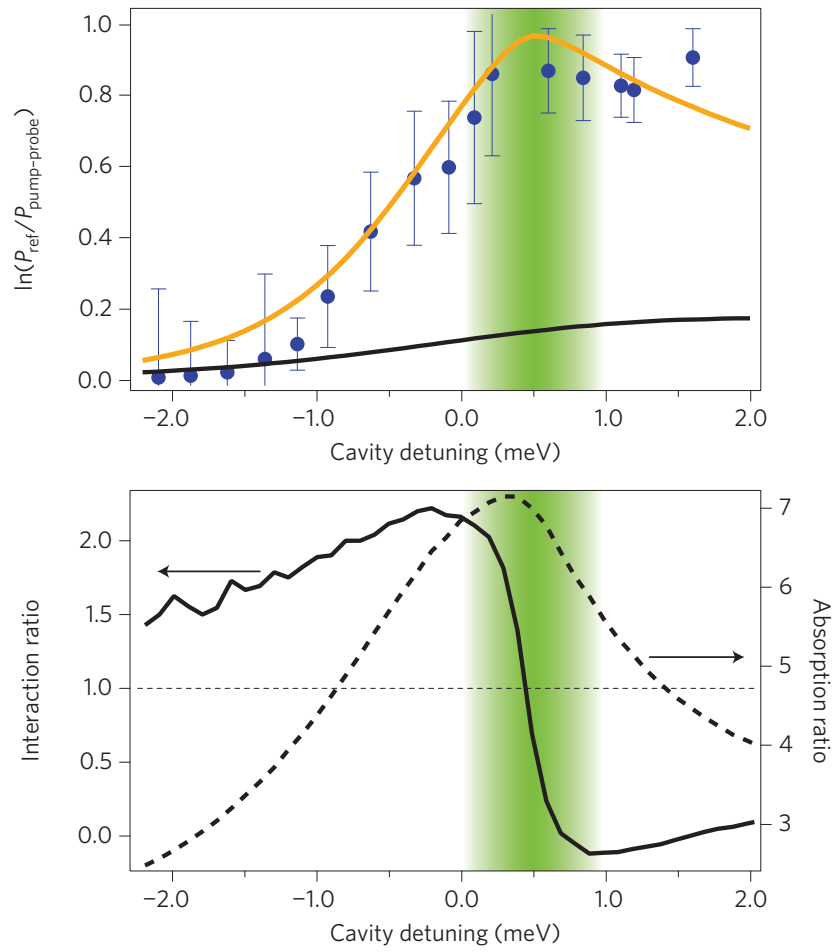

Figure 2 | Polaritonic Feshbach resonance: experimental manifestations. a, Energy shifts of the pump-probe spectrum as a funciton of cavity detuning. $\mathbf{b}, \ln \left(P_{\text {ref }} / P_{\text {pump-probe }}\right)$ as a function of cavity detuning, where $P_{\text {ref }}$ and $P_{\text {pump-probe }}$ represent the amplitude of the lower polariton resonance without and with pump excitation, respectively. The blue circles are experimental results, orange and black lines show numerical simulations with and without polariton-biexciton coupling, respectively. c, Interaction (solid curve; left axis) and absorption (dashed curve; right axis) ratios as a function of the cavity detuning. They result from the ratio between the orange and black lines extracted from Fig. 2a,b respectively. Fitting parameters are summarized in the simulation section of the Methods. The fitted density of polaritons is $n_{p}=n_{0}$. The green region corresponds to the energy range where the two-polariton energy crosses the biexciton state energy: $E_{\mathrm{LP} \uparrow}+E_{\mathrm{LP} \downarrow} \leftrightarrow E_{\mathrm{BX}}$ (Fig. 1c). Error bars represent \pm 3 standard deviations.

We then extract the energy shifts and the amplitude variations of the polariton resonance between the calculated $n_{\mathrm{p}}=0$ and $n_{\mathrm{p}} \neq 0$ probe transmission spectra as a function of cavity detuning. In Fig. 2a,b, the orange lines represent, respectively, the polariton energy shift and amplitude variations obtained by the numerical calculations for $n_{\mathrm{P}}=n_{0}$. Here, $n_{0}$ is a normalized polariton population density corresponding to the experimentally extracted polariton density of $5.1 \times 10^{10}$ polaritons $\mathrm{cm}^{-2}$. The other fitting parameters are summarized in the simulation section of Methods. Note that the calculated results overlap very well with the
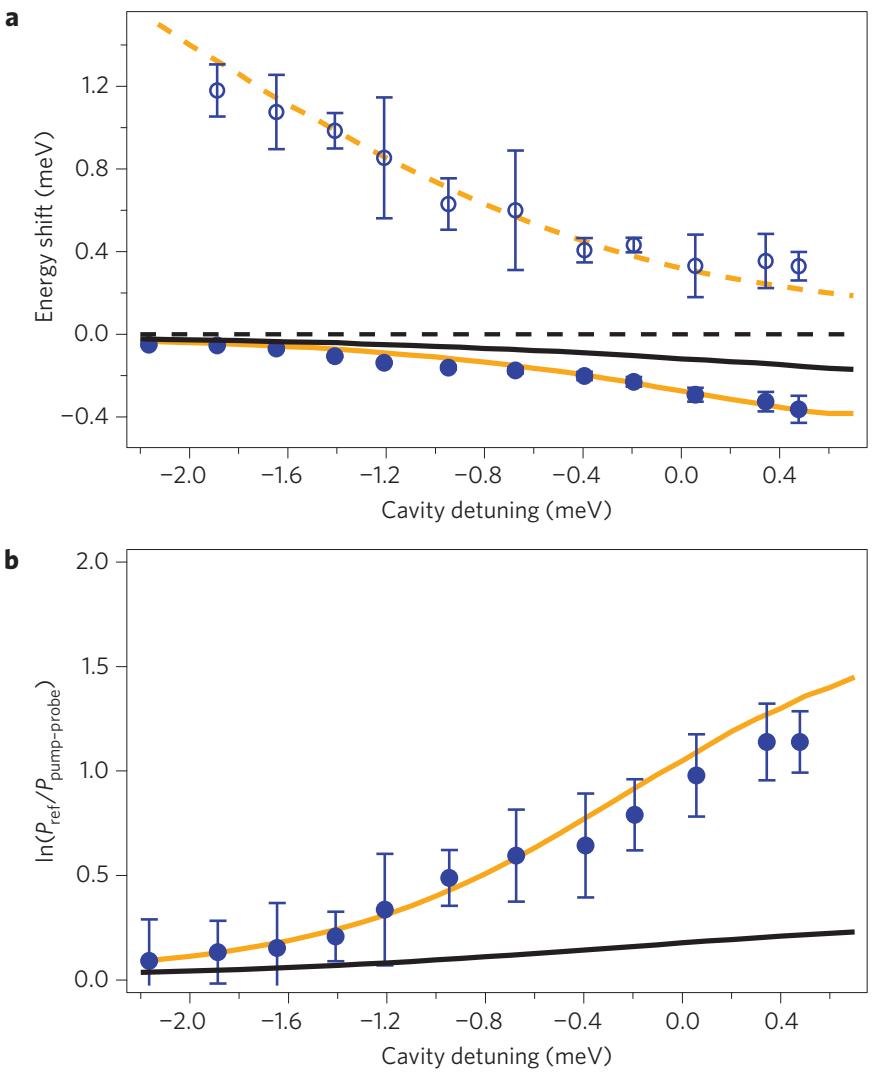

Figure 3 | Higher-density polaritonic Feshbach effect. a, Energy shift of the pump-probe spectrum as a function of cavity detuning for a polariton pump density of $1.6 \times 10^{11}$ polaritons $\mathrm{cm}^{-2}$. For higher pump intensities, measurements are limited below 0.5 meV cavity detuning to avoid a strong to weak coupling transition, which would render the polariton picture no longer appropriate. $\mathbf{b}, \ln \left(P_{\text {ref }} / P_{\text {pump-probe }}\right)$ for the lowest polariton branch as a function of the cavity detuning. Orange and black lines, respectively, represent numerical simulations with and without polariton-biexciton coupling obtained with a density $n_{\mathrm{p}}=2.44 n_{0}$. Error bars represent \pm 3 standard deviations.

experimental plots, showing a very good agreement between theory and experiment. The plotted black lines correspond to the calculated energy shift and amplitude variations using the same parameters, but without the polariton-biexciton scattering term $\left(g_{\mathrm{BX}}=0\right)$. Here, only the background attractive anti-parallel spin polariton interaction exists. Note that even without the biexcitonic effect, the mean-field polariton energy shift and amplitude variations depend on the cavity detuning. In Fig. 2a (black curve), the polariton energy redshift increases according to the fourth power of the excitonic Hopfield coefficient ${ }^{23,25}$. The experimental results, however, strongly deviate from this behaviour when the biexciton resonance is approached. This result evidences the fact that the prominent enhancement of the energy redshift, as well as the change of sign of the interaction from attractive to weak repulsive, can only be accounted for by the polariton-biexciton coupling, confirming the polaritonic Feshbach resonance.

Concerning the change of amplitude of the polariton field, there exist two contributions. The first contribution is an effective positive increase of cavity detuning induced by the polariton energy redshift due to the background attractive interaction ${ }^{26}$, which reduces the photonic component of the polariton and therefore imply a decrease of the amplitude of the polariton resonance (black line in Fig. 2b). This contribution alone evidently does not reproduce the experimental results. The second contribution is the decay of two polaritons with anti-parallel spins into the biexciton channel as a 
result of polariton-biexciton coupling. As is clearly shown in Fig. 2b (orange curve), to reproduce accurately the large decrease of the polariton resonance amplitude the polariton spin-up + polariton spin-down $\rightarrow$ biexciton scattering process should be introduced in the model.

We plot in Fig. 2c interaction and absorption ratios extracted from Fig. 2a,b respectively. Those ratios result from the total contribution (orange curves) divided by the background contribution (black curves). These two ratios evidence the usual behaviour of a scattering resonance. The absorption ratio (dashed curve) behaves as the expected scattering resonance profile due to the two-body loss process of lower polaritons when crossing the molecular state. The interaction ratio (solid curve) exhibits a dispersive shape of the scattering length, showing a large variation in terms of the sign and amplitude of the interaction strength crossing the biexciton resonance. Analogous observations are reported by Theis et al. ${ }^{4}$ in a ${ }^{87} \mathrm{Rb}$ condensate in optical Feshbach resonance.

This model, in which we consider the interaction of the two anti-parallel spin polaritons with the molecular biexciton state, reproduces the experimental results very well. This is the resonant interaction term that governs a Feshbach resonance. In the present model, it is important to note that the coupling between polaritons and biexcitons, the coupling term $g_{\mathrm{BX}} \sqrt{n_{X}}$, depends not only on the constant $g_{\mathrm{BX}}$ but also on the excitonic content of polariton population $\sqrt{n_{X}}$. At low pump power, the pump polariton density is small and, consequently, this coupling term is weak. In this perturbative regime, the comparison between simulations and experiments shows excellent qualitative and quantitative agreement, demonstrating unambiguously the polaritonic Feshbach resonance.

We now turn to the investigation of the polaritonic Feshbach resonance in a system with a larger polariton population. We perform pump and probe measurements as a function of the cavity detuning using higher pump intensities to increase the polariton population. As the pump power is increased, a new resonance shows up at higher energy than the polariton resonance (Fig. 1e). In Fig. 3 we plot, for a polariton density of $1.6 \times 10^{11}$ polaritons $\mathrm{cm}^{-2}$ generated by the pump, the energy shift of both polariton resonances and the amplitude variation of the lower energy polariton resonance as a function of the cavity detuning.

When the cavity detuning brings the two-polariton state energy close to the biexciton bound state energy then both resonances show an anti-crossing behaviour (Fig. 3a). The associated energy shifts obtained from the numerical simulations using the same parameters as for the lower power regime, but now with $n_{\mathrm{p}}=2.44 n_{0}$, are shown as full and dashed orange lines for the lower and higher energy resonances, respectively. The numerical results show remarkable quantitative agreement with the experimental results. We also show the numerical result obtained without considering the polariton-biexciton coupling $\left(g_{\mathrm{BX}}=0\right)$ as solid black line, which clearly deviates from the experimental data. In this high-density regime, the appearance of a new resonance and the anti-crossing behaviour evidence the strong polariton-biexciton coupling. It is important to note that, to reach this strong coupling regime, the polariton-biexciton coupling energy $g_{\mathrm{BX}} \sqrt{n_{X}}$ should be comparable to the linewidths of the involved states. In our system, the polariton-biexciton coupling strength is directly controlled by the density of polaritons through the pump intensity. Then, for the pump intensity used in Fig. 3, the polariton-biexciton coupling energy is of the same order as the biexciton linewidth. Indeed, in this regime, the polaritonic Feshbach resonance shows an anticrossing between these two states in addition to the change of the polariton interaction.

Moreover, as the spin-up polariton population is increased, the two spin-up spin-down polariton correlation is enhanced, which favours the biexciton creation. In Fig. $3 b$, we plot the amplitude variations of the lower energy resonance shown in Fig. 3a. Enhancement of the amplitude variation with pump intensity and consequent increased losses with pump polariton population are evidenced. This supports the enhancement of the biexciton formation for the larger polariton population. The very good agreement between experimental and theoretical values corroborates our interpretation as a polaritonic Feshbach resonance.

All these results together demonstrate two different regimes of polaritonic Feshbach resonance. First, in the low-density regime, where polaritons and biexcitons show small interactions, the polariton-biexciton coupling energy cannot overcome the damping rate of the biexcitons $2 g_{\mathrm{BX}} \sqrt{n_{X}}<\gamma_{B}$. Therefore, in this weak interaction regime, the manifestation of the Feshbach resonance exhibits a dispersive shape. In contrast, in the strong interaction regime $2 g_{\mathrm{BX}} \sqrt{n_{X}} \sim \gamma_{B}$, polaritons and biexcitons states manifest an anticrossing. The splitting energy, proportional to twice the polariton-biexciton coupling term, increases for much higher pump intensities (not shown). As a result, the polariton population governs the polariton-biexciton coupling strength, which allows for the polaritonic Feshbach resonance to be tuned from the weakly to strongly interacting regime.

\section{Methods}

Our study is performed on a high-quality III-V microcavity. A single $8 \mathrm{~nm}$ $\mathrm{In}_{0.04} \mathrm{Ga}_{0.96}$ As quantum well is sandwiched between a pair of GaAs/AlAs distributed Bragg-reflectors (DBRs). The exciton energy of the quantum well is $1.4866 \mathrm{eV}$ and the Rabi splitting at zero detuning between the cavity and exciton energy is $3.26 \mathrm{meV}$ (ref. 27). The measurements are performed at a temperature of approximately $4 \mathrm{~K}$. We employ a counter-circular polarization configuration to investigate the polariton-polariton interaction with anti-parallel spins. To

demonstrate the Feshbach resonance on the spinor polariton scattering, we carry out polarization-resolved pump-probe spectroscopy in transmission. We employ a heterodyne measurement technique ${ }^{12,28}$, which markedly increases the signal to noise ratio. It allows one to resolve small probe beam energy shifts and to use a degenerate beam configuration. Figure 1b,c shows the schematics of our pump-probe experiment. The sample is resonantly excited at $k=0$ with a $\sigma$ circularly polarized pump pulse to generate a spin-up polariton population and probed at $k=0$ using a counter-circularly polarized probe pulse. Because the cavity spacer layer is wedged, we tune the resonance energy of the cavity by moving the laser spot over the sample. The biexciton binding energy $E_{\text {bix }}$ is of the same order as the Rabi splitting. It is then straightforward to bring the energy of a two-lower-polariton state in resonance with the biexciton state by changing the cavity-exciton detuning (Fig. 1a,c). We repeat the experiment for different cavity detunings and different pump powers.

The laser source is a $125 \mathrm{fs}$ pulse with a repetition rate of $80 \mathrm{MHz}$, which is separated into three pulses: pump, probe and reference. The pump spot size $\left(2,200 \mu \mathrm{m}^{2}\right)$ is larger than probe size $\left(250 \mu \mathrm{m}^{2}\right)$ to ensure the study of a uniform pump polariton density. We fixed the probe intensity at approximately one third the pump intensity in the low-density regime (Fig. 2). Then, increasing the pump intensity, the pump/probe density ratio is even larger. The centre frequency of the laser is set between the lower and upper polaritons. In our measurement we varied the LP energy only within $2 \mathrm{meV}$. As the laser spectrum is about $14.6 \mathrm{meV}$ wide, the laser intensity is considered to be constant for all laser detunings. Both probe and reference beams are detected by a spectrometer and the spectrum of the probe pulse is numerically reconstructed.

Simulation. The biexciton energy is determined as $\varepsilon_{B}=\varepsilon_{X}-E_{\text {bix }} / 2$, with $E_{\text {bix }}$ being the biexciton binding energy. For the numerical calculation of Figs 2 and 3 we use the following parameters: the biexciton binding energy $E_{\mathrm{bix}} / 2=1.5 \mathrm{meV}$, a biexciton linewidth $\gamma_{B}=1.1 \mathrm{meV}$. For the polariton-biexciton coupling, the exciton-exciton scattering term is set to $g_{\mathrm{BX}}=0.36 \mathrm{meV} / \sqrt{n_{0}}$. The background interaction is $U_{\mathrm{bg}}=-0.18 \mathrm{meV} / n_{0}$, where $n_{0}$ is the normalized density of spin-up polaritons. The other parameters are the photon linewidth $\gamma_{C}=0.3 \mathrm{meV}$, exciton linewidth $\gamma_{X}=0.6 \mathrm{meV}$ and Rabi coupling $\Omega_{X}=1.63 \mathrm{meV}$. Note that the same parameter values are used for all experiments, except for the polariton density $n_{P}$, which is adjusted when the pump density is changed. The excitonic density $n_{X}$ is expressed as a function of the polariton density $n_{P}$ through the expression:

$$
n_{X}=\frac{n_{P}}{2}\left(1+\frac{\delta}{\sqrt{\delta^{2}+4 \Omega_{X}^{2}}}\right)
$$

where $\delta$ is the exciton-cavity detuning. Polariton density is estimated through the density of the excitonic component. In the higher-density experiment, we observe 
a strong to weak coupling transition at the cavity detuning $0.87 \mathrm{meV}$. This means that, at this cavity detuning, the excitonic component of polaritons reaches the exciton saturation density $n_{\text {Xsat }}$, which is given by $n_{\text {Xsat }}=7 / 16 \pi a_{2 D}^{2} \approx 1 \times 10^{11} \mathrm{~cm}^{2}$ (ref. 29) for a Bohr radius of $12 \mathrm{~nm}$ in a single InGaAs quantum well ${ }^{30}$. The polariton density can then be estimated using (1). To facilitate the extraction of the new upper resonance energy position in the large polariton population experiment (orange dashed line Fig. 3), we force $\gamma_{b x}=0$ in the model to enhance the peak transmission. Note that this procedure does not affect the resonance energy positions.

Received 10 July 2013; accepted 19 May 2014; published online 22 June 2014

\section{References}

1. Inouye, S. et al. Observation of Feshbach resonances in a Bose-Einstein condensate. Nature 392, 151-154 (1998).

2. Bloch, I., Dalibard, J. \& Zwerger, W. Many-body physics with ultracold gases. Rev. Mod. Phys. 80, 885-964 (2008).

3. Chin, C., Grimm, R., Julienne, P. \& Tiesinga, E. Feshbach resonances in ultracold gases. Rev. Mod. Phys. 82, 1225-1286 (2010).

4. Theis, M. et al. Tuning the scattering length with an optically induced Feshbach resonance. Phys. Rev. Lett. 93, 123001 (2004).

5. Donley, E. A. et al. Dynamics of collapsing and exploding Bose-Einstein condensates. Nature 412, 295-299 (2001).

6. Greiner, M., Regal, C. \& Jin, D. S. Emergence of a molecular Bose-Einstein condensate from a Fermi gas. Nature 426, 537-540 (2003).

7. Kasprzak, J. et al. Bose-Einstein condensation of exciton polaritons. Nature 443, 409-414 (2006).

8. Amo, A. et al. Superfluidity of polaritons in semiconductor microcavities. Nature Phys. 5, 805-810 (2009).

9. Lagoudakis, K. G. et al. Quantized vortices in an exciton-polariton condensate. Nature Phys. 4, 706-710 (2008).

10. Utsunomiya, S. et al. Observation of Bogoliubov excitations in exciton-polariton condensates. Nature Phys. 4, 700-705 (2008).

11. Kohnle, V. et al. From single particle to superfluid excitations in a dissipative polariton gas. Phys. Rev. Lett. 106, 255302 (2011).

12. Kohnle, V. et al. Four-wave mixing excitations in a dissipative polariton quantum fluid. Phys. Rev. B 86, 064508 (2012).

13. Savvidis, P. G. et al. Angle-resonant stimulated polariton amplifier. Phys. Rev. Lett. 84, 1547-1550 (2000).

14. Baas, A., Karr, J. P., Eleuch, H. \& Giacobino, E. Optical bistability in semiconductor microcavities. Phys. Rev. A 69, 023809 (2004).

15. Paraïso, T. K., Wouters, M., Leger, Y., Morier-Genoud, F. \& Deveaud-Pledran, B. Multistability of a coherent spin ensemble in a semiconductor microcavity. Nature Mater. 9, 655-660 (2010).

16. Amo, A. et al. Exciton-polariton spin switches. Nature Photon. 4, 361-366 (2010).

17. Cerna, R. et al. Ultrafast tristable spin memory of a coherent polariton gas. Nature Commun. 4, 2008 (2013).

18. Carusotto, I., Volz, T. \& Imamoğlu, A. Feshbach blockade: Single-photon nonlinear optics using resonantly enhanced cavity polariton scattering from biexciton states. Europhys. Lett. 90, 37001 (2010).
19. Borri, P., Langbein, W., Woggon, U., Jensen, J. R. \& Hvam, J. M. Biexcitons or bipolaritons in a semiconductor microcavity. Phys. Rev. B 62, R7763-R7766 (2000).

20. Saba, M. et al. Crossover from exciton to biexciton polaritons in semiconductor microcavities. Phys. Rev. Lett. 85, 385-388 (2000).

21. Vladimirova, M. et al. Polariton-polariton interaction constants in microcavities. Phys. Rev. B 82, 075301 (2010).

22. Baars, T., Dasbach, G., Bayer, M. \& Forchel, A. Biexciton states in semiconductor microcavities. Phys. Rev. B 63, 165311 (2001).

23. Wouters, $M$. Resonant polariton-polariton scattering in semiconductor microcavities. Phys. Rev. B 76, 045319 (2007).

24. Ivanov, A. L., Hasuo, M., Nagasawa, N. \& Haug, H. Two-photon generation of excitonic molecules in $\mathrm{CuCl}$ : An exactly solvable bipolariton model and high-precision experiments. Phys. Rev. B 52, 11017-11033 (1995).

25. Ciuti, C., Schwendimann, P., Deveaud, B. \& Quattropani, A. Theory of the angle-resonant polariton amplifier. Phys. Rev. B 62, R4825-R4828 (2000).

26. Vladimirova, M. et al. Polarization controlled nonlinear transmission of light through semiconductor microcavities. Phys. Rev. B 79, 115325 (2009).

27. Stanley, R.P., Houdré, R., Oesterle, U., Gailhanou, M. \& Illegems, M. Ultrahigh finesse microcavity with distributed Bragg reflectors. Appl. Phys. Lett. 65, 1883-1885 (1994)

28. Hall, K. L., Lenz, G., Ippen, E. P. \& Raybon, G. Heterodyne pump-probe technique for time-domain studies of optical nonlinearities in waveguides. Opt. Lett. 17, 874-876 (1992).

29. Rochat, G., Ciuti, C., Savona, V., Piermarocchi, C. \& Quattropani, A. Exitonic Bloch equations for a two-dimensional system of interacting excitons. Phys. Rev. B 61, 13856-13862 (2000).

30. Grassi Alessi, M. et al. Competition between radiative decay and energy relaxation of carriers in disordered $\operatorname{In}_{x} \mathrm{Ga}_{1-x} \mathrm{As} / \mathrm{GaAs}$ quantum wells. Phys. Rev. B 61, 10985-10993 (2000).

\section{Acknowledgements}

We thank I. Carusotto for fruitful discussions. The present work is supported by the Swiss National Science Foundation under project No 135003, the Quantum Photonics National Center of Competence in Research No 115509 and by the European Research Council under project Polaritonics contract No 219120. The POLATOM network is also acknowledged.

\section{Author contributions}

N.T. and S.T. contributed to the experiment. N.T. performed the measurements and the data processing. M.W. developed the theoretical model. N.T. and S.T. performed the simulations. N.T., S.T. and M.T.P-O. performed data analysis. S.T. and M.T.P-O. wrote the paper. M.T.P-O. and B.D. supervised the whole project. All authors contributed to numerous discussions and revised the manuscript.

\section{Additional information}

Supplementary information is available in the online version of the paper. Reprints and permissions information is available online at www.nature.com/reprints. Correspondence and requests for materials should be addressed to N.T.

\section{Competing financial interests}

The authors declare no competing financial interests. 